\title{
Preliminary Study on the Feasibility of Early Feeding After General Anesthesia and Lumbar Spine Surgery in Middle-aged and Elderly Patients
}

\author{
Yuanqun Zou ${ }^{1}$, Xiaorong Zhang ${ }^{1}$, Yanfei $\mathrm{Li}^{1}$, Cuiqing Liu ${ }^{2, *}$ \\ ${ }^{1}$ Department of Orthopaedics, The First Affiliated Hospital of Jinan University, Guangzhou, China \\ ${ }^{2}$ Department of Primary Surgery, The First Affiliated Hospital of Jinan University, Guangzhou, China
}

Email address:

cuiqing.liu@yahoo.com (Cuiqing Liu)

${ }^{*}$ Corresponding author

\section{To cite this article:}

Yuanqun Zou, Xiaorong Zhang, Yanfei Li, Cuiqing Liu. Preliminary Study on the Feasibility of Early Feeding After General Anesthesia and Lumbar Spine Surgery in Middle-aged and Elderly Patients. American Journal of Nursing Science. Vol. 8, No. 6, 2019 , pp. 346-350. doi: 10.11648/j.ajns.20190806.19

Received: November 7, 2019; Accepted: November 28, 2019; Published: December 11, 2019

\begin{abstract}
To investigate the feasibility of early feeding after lumbar spine surgery in middle-aged and elderly patients. Seventy middle-aged and elderly patients with lumbar disc herniation, lumbar spinal stenosis or lumbar spondylolisthesis after treatments of single-segment lumbar discectomy + spinal canal decompression + interbody fusion after total anesthesia with pedicle screw fixation have been selected from January 2018 to May 2019. They were randomly divided into the experimental group and the control group. The experimental group had semi-liquid diet and water at 2 hours after operation. The control group had semi-liquid diet and water at 6 hours after operation; the first exhaust/defecation time, the incidence of nausea/vomiting after eating, and the incidence of postoperative bloating were compared between the two groups. The first exhaust/defecation time of the experimental group was earlier than the control group $(P<0.05)$, and the incidence of postoperative abdominal distension was lower in the experimental group $(P<0.05)$. There was no significant difference in the rate of postoperative nausea/vomiting occurrence in the two groups. $(P>0.05)$. The middle-aged and elderly patients undergoing general anesthesia for lumbar spine surgery should have faster deflation/defecation within 2 hours postoperatively eating, than 6 hours, to reduce the incidence of postoperative abdominal distension without increasing the incidence of nausea/vomiting adverse reactions.
\end{abstract}

Keywords: General Anesthesia, Lumbar Surgery, Middle-aged and Elderly Patients, Early Feeding

\section{Introduction}

The economic level and scientific technology have been continuously improved with the development of society; people's lifestyle has undergone major changes. The previous large amount of manual labor was gradually undertaken by machines and artificial intelligence, ways of life and work gradually became more manual labor. The "dynamic mode" has been transformed into a "static mode" of sedentary, long-time standing and lack of physical activity. The degenerative changes brought about by the "static" lifestyle have also become lower in age. Among them, the degenerative changes of the spine are particularly prominent, and it has become one of the major diseases that plague people, especially middle-aged and elderly people.
Spinal degeneration mainly includes cervical and lumbar degenerative changes, while lumbar degenerative changes mainly include lumbar disc herniation, lumbar spinal stenosis and lumbar spondylolisthesis. According to the World Health Organization, more than $15 \%$ of the world's population suffer from lumbar disc herniation, and the incidence is on the rise [1].

For patients with general anesthesia for non-gastrointestinal surgery, it is generally considered that adults were fasting 8-12 hours and stop drinking 4 hours before surgery to prevent asphyxia or aspiration pneumonia due to anesthesia or intraoperative vomiting. If necessary, gastrointestinal decompression is feasible [2]. However, there are many different opinions about the after-surgery eating time. Even the latest edition of the Surgery (9th 
Edition) textbook of the People's Health Publishing House does not clearly explain this. It is generally accepted that after fasting for 6 hours or after anal exhaust. However, prolonged fasting can easily bring a series of physical and psychological discomforts and complications to the patient, which in turn affects the effect of surgery and postoperative recovery. Therefore, we investigated the possibility of eating early in the middle-aged and elderly people after general anesthesia by observing the postoperative feeding time and the effect on postoperative anal exhaust/defecation as well as the incidence of nausea/vomiting and postoperative bloating after eating.

\section{Materials and Methods}

\subsection{General Information}

Seventy middle-aged and elderly patients in our hospital with lumbar disc herniation, lumbar spinal stenosis or lumbar spondylolisthesis after treatments of single-segment lumbar discectomy + spinal canal decompression + interbody fusion after total anesthesia with pedicle screw fixation have been selected from January 2018 to May 2019, including 36 male patients and 34 female patients, were randomly divided into the experimental group and the control group, including 17 female patients and 18 male patients. The experimental group began to eat 2 hours after the operation, and the control group began to eat 6 hours after the operation. The average age of the patients in the experimental group was (57.60 5.24) years old, and the average age of the control group patients was $(57.97 \pm 5.18)$ years old. There was no significant difference between the two groups $(P>0.05)$. All the patients were informed and consented by the study and met the standards of the hospital ethics committee.

\subsection{Inclusion Criteria}

(1) Single-segment, single lumbar vertebrae (lumbar disc herniation, lumbar spinal stenosis or lumbar spondylolisthesis) consistent with clinical data and imaging data; (2) Age between 50 and 65 years old; (3) Preoperative 15 days of stool as usual (about 1-2 times, stool formation, color yellow / dark yellow, soft, volume), urine as usual.

\subsection{Exclusion Criteria}

(1) With history of chronic diarrhea or constipation; (2) Acute gastrointestinal symptoms 15 days before surgery; (3) Previous viral hepatitis, peptic ulcer, gastrointestinal bleeding, digestive tract perforation, cirrhosis, cholecystitis, pancreatitis, appendicitis History of digestive system such as peritonitis; (4) With history of previous abdominal surgery; (5) With history of acute/malignant hypertension, diabetes, coronary heart disease, malignant tumor and mental illness; (6) Obesity and thin body type; (7) Previous food and medicine history of allergies; (8) Previous history of general anesthesia or lumbar surgery.

\subsection{Surgery and Anaesthesia}

All patients were sent to the surgical center of our hospital for tracheal intubation underwent single-segment lumbar discectomy + spinal canal decompression + interbody fusion and posterior pedicle screw fixation. The operation started immediately after the successful anesthesia, and ended within 2.5 hours. After the operation, the anesthesia was resuscitated in the recovery room of our hospital. All patients were awakened within 0.5 hours, and returned to our orthopaedic ward within 5 minutes after waking up with recording the time of eating.

\subsection{Postoperative Drug Use}

All patients were treated with "injection with cefazolin sodium pentahydrate" to prevent infection, "omeprazole for injection" to protect stomach, "ketose tromethamine injection" and other symptomatic supportive treatment.

\subsection{Eating Methods and Contents}

The experimental group began to eat 2 hours after the operation, and the control group began to eat at 6 hours after the operation; the body position at the time of eating and 30 minutes after eating was supine, and the bed was shaken $30^{\circ}$; Southern rice porridge $100 \mathrm{~g}$ and warm water $100 \mathrm{ml}$ was made in the canteen of our hospital. Complete eating within 10min.

\subsection{Observation Indicators}

The first venting/defecation time after surgery, the number of cases of nausea/vomiting and bloating after surgery.

\subsection{Statistical Methods}

Data were processed using spss 19.0 statistical software. The measurement data $(\bar{x} \pm S)$ were analyzed by independent $t$-test, and the count data (\%) were tested by $x^{2}$ test of four-table data; $P<0.05$ was considered statistically significant.

\section{Results}

The first exhaust time of the experimental group was $(0.91 \pm 0.61) \mathrm{d}$, which was earlier than the first exhaust time $(1.71 \pm 0.62) \mathrm{d}$ after the control. The difference was statistically significant $(P<0.05)$ (Table 1); There was no postoperative vomiting response in all the patients tested. There was no significant difference in the incidence of nausea after eating between the experimental group and the control group $(P>$ 0.05 ) (Table 2). The incidence of postoperative abdominal distension in the experimental group was $8.6 \%$. The incidence of postoperative abdominal distension was $28.6 \%$ in the test group, and the difference was statistically significant $(P<0.05)$ (Table 3). 
Table 1. Comparison of first exhaust/defecation time in two groups.

\begin{tabular}{lllll}
\hline Group & Eating time (h) & Exhaust/bill time (d) & T value & P value \\
\hline Test group & 2 & $0.91 \pm 0.61$ & \multirow{2}{*}{5.424} & 0.000 \\
Control group & 6 & $1.71 \pm 0.62$ & & \\
\hline
\end{tabular}

Table 2. Comparison of the incidence of nausea after eating in both groups (\%).

\begin{tabular}{lllll}
\hline Group & Nausea response & No Nausea response & $\overline{\boldsymbol{x}}$ & $\boldsymbol{P}$ value \\
\hline Test group & $4(11.4)$ & $31(88.6)$ & 1.609 & 0.205 \\
Control group & $8(22.9)$ & $27(77.1)$ & 1.6 & \\
\hline
\end{tabular}

Table 3. Comparison of the incidence of abdominal distension after eating in two groups (\%).

\begin{tabular}{lllll}
\hline Group & Bloating & No Bloating & $\overline{\boldsymbol{x}}$ & \multicolumn{1}{c}{ Pvalue } \\
\hline Test group & $3(8.6)$ & $32(91.4)$ & 4.629 & \multirow{2}{*}{0.031} \\
Control group & $10(28.6)$ & $25(71.4)$ & & \\
\hline
\end{tabular}

\section{Discussion}

\subsection{Overview of Lumbar Decompression + Fusion + Internal Fixation Surgery}

Lumbar degenerative diseases generally refer to a series of symptoms caused by compression of nerve roots or cauda equina due to lumbar disc degeneration, lumbar vertebrae and ligament soft tissue hyperplasia, including lumbar disc herniation, lumbar spinal stenosis and lumbar spondylolisthesis. They are common diseases and frequently-occurring diseases in the field of spinal surgery and the most common causes of low back pain. It can cause damage to nerve function, which in turn causes muscle strength and paresthesia of both lower limbs, thus affecting patients' daily routines [3]. In China, the incidence of lumbar degenerative diseases is gradually increasing and the age is lower [4].

The treatment of lumbar degenerative diseases generally includes conservative treatment and surgical treatment. Conservative treatments include bed rest, lumbar traction, drug therapy, and physical therapy. For patients with short course and mild symptoms, they can play a role in relieving symptoms, but it is easy to recurrent onset. For patients with severe symptoms, which have seriously affected daily life and rest, poor or ineffective conservative treatment, with persistent nerve compression symptoms, surgical treatment should be performed as soon as possible [2].

Lumbar discectomy + spinal canal decompression + interbody fusion + posterior pedicle screw fixation is one of the most classic surgical procedures in the field of spinal surgery, widely used in the treatment of lumbar degenerative diseases [5]. Compared with the minimally invasive techniques that have emerged in recent years, such as the technique of intervertebral foramen, the technique of percutaneous pedicle screwing, the technique of lower lumbar fusion, etc., it has a complete decompression, the fusion effect is exact, and the internal fixation is relatively simple and reliable. In addition to the $\mathrm{C}$-arm X-ray machine, no special equipment is required and the cost is low. Therefore, this procedure is still favored by many spine surgeons and patients.

\subsection{The Adverse Effects of Long-term Fasting During the Perioperative Period on the Body}

Long-term sense of hunger, thirsty and fear of surgery, nervousness can cause hunger, dizziness, headache, irritability, restlessness, hypoglycemia [6], and even reduce the patient's treatment compliance, which is the effect of surgery, surgery Post-rehabilitation and mental health are very unfavorable [7], even may affect the patient's surgical satisfaction and post-operative follow-up compliance [8].

Long-term hunger and surgical stress can increase the secretion of tight adrenaline and glucagon, activate glycogen phosphorylase, and promote hepatic glycogenolysis. At the same time, adrenal cortex hormone and glucagon can strengthen gluconeogenesis; tight glandular corticosteroids and growth hormone reduce the utilization of sugar by peripheral tissues. The secretion of these hormones can increase blood sugar [9]. Studies have shown that long-term banned diet and intraoperative trauma can lead to insulin resistance, which further increases blood glucose $[10,11]$, affecting the recovery of the postoperative body.

Prolonged fasting is easy to cause the body's water and electrolyte balance and hemodynamic disorder, and the trauma of the surgery itself increases the consumption of protein, sugar, etc., which reduces the immune function of the body, and the gastrointestinal dysfunction can increase the bacterial translocation. Risk, thereby increasing the risk of intraoperative and postoperative infections $[12,13]$. At the same time, the stress state during surgery and the negative nitrogen balance during perioperative period can increase the incidence of stress ulcers [14] and aggravate the original chronic diseases (hypertension, diabetes, heart disease, etc.) [6].

Prolonged fasting can easily cause gastrointestinal dysfunction [12, 13], plus anesthetic drugs can weaken gastrointestinal motility, which is not conducive to the recovery of postoperative gastrointestinal function [15], leading to postoperative nausea, vomiting, bloating After adverse reactions, severe cases may have postoperative 
intestinal paralysis. Therefore, under the premise of ensuring postoperative safety, it is necessary to shorten the postoperative feeding time.

\subsection{Analysis of the Feasibility of Early Feeding After Tracheal Intubation}

Traditional lumbar discectomy + spinal canal decompression + interbody fusion + posterior pedicle screw fixation because the surgical position is prone position, the operation plane is higher, the operation time is longer (single segment operation time is generally $2-3 \mathrm{~h}$ ), the general anesthesia used is general anesthesia for tracheal intubation. As mentioned above, the current accepted time for the postoperative feeding time is that after fasting for 6 hours or after anal exhaust, the orthopedics department of our hospital has always followed this method. However, this will result in a patient's perioperative fasting time of at least about 17 hours (for example, anesthesia, surgery, and postoperative resuscitation for 3 hours). If it is followed by surgery, the fasting time can be if 20 hours or more. During the time, the patient can maintain the required energy and internal environment balance through intravenous rehydration or parenteral nutrition, but this cannot promote the recovery and maintenance of perioperative intestinal function.

In this trial, we tried to shorten the postoperative fasting time to 2 hours. Compared with the patients who were fasted for 6 hours after surgery, it was found that eating 2 hours after surgery can shorten the anal exhaust/defecation time and reduce the occurrence rate of postoperative abdominal distension. It may be due to the early expansion of the food, which can stimulate the dilatation of the stomach. The vagus-vaginal reflex and the local reflex of the inner plexus of the stomach wall to cause the stomach movement to strengthen. And some chemical components in the food can cause the stomach pyloric $G$ cells release gastrin, which promotes the movement of the stomach. At the same time, the movement of the small intestine is mainly regulated by the myenteric plexus, and the mechanical and chemical stimulation of the intestinal mucosa by the chyme can enhance the movement by local reflex [16]. Above gastrointestinal physiological changes are beneficial to promote the recovery of gastrointestinal function and shorten the time of exhaust and defecation. In recent years, studies have shown that early feeding after general anesthesia does not increase the incidence of postoperative complications such as nausea, vomiting, and abdominal distension [17], which is basically consistent with our results. In this paper, we found that eating 2 hours after surgery did not increase the incidence of nausea/vomiting. Because of the early release of hunger and thirst, it has a positive effect on improving patient comfort, surgical satisfaction, postoperative treatment compliance and improving postoperative adverse emotions to make postoperative patients get to health faster.

\section{Conclusion}

Middle-aged and elderly patients undergoing general anesthesia for lumbar spine surgery should have faster deflation/defecation within 2 hours postoperatively eating than 6 hours. It reduces the incidence of postoperative abdominal distension without increasing the incidence of nausea/vomiting adverse reactions. With early feeding after general anesthesia and lumbar spine surgery, middle-aged and elderly patients get a beneficial recovery.

\section{Acknowledgements}

The authors declare no conflict of interest.

\section{References}

[1] Lee SE, Jahng TA, Kim HJ. Decompression and nonfusion dynamic stabilization for spinal stenosis with degenerative lumbar scoliosis: Clinical article [J]. J Neurosurg Spine, 2014; 21 (4): 585-594.

[2] Wu Mengchao WZ, Wu Yuhan, et al. Surgery (9th Edition) [M]. [J]. Beijing: People's Medical Publishing House, 2018: 91-98.

[3] Meng Ningbo YZ, Sun Jianmin. A comparative study of TLIF and open TLIF in the treatment of lumbar degenerative diseases with quadrant rant minimally invasive system [J]. Chinese Journal of Modern Medicine, 2014; 24 (28): 90-93.

[4] Yin Gang ZZ, Sun Zhi, et al. Risk factors for lumbar intervertebral disc herniation in Chinese population: a case-control study [J]. Spine, 2009; 34 (25): 918-922.

[5] Fan Haiquan YY, Zeng Xiangjia, et al. Treatment of lumbar degenerative diseases with lumbar interbody fusion [J]. Journal of Spinal Surgery, 2007; 5 (1): 55-57.

[6] Xu Haiying SW. Study on shortening preoperative fasting and banned time in patients undergoing elective surgery [J]. Journal of Nurses Training, 2010; 25 (2): 109-111.

[7] Wei Q. Progress in preoperative fasting and banned drinking time in patients undergoing elective surgery $[\mathrm{J}]$. Chinese Journal of Nursing, 2014; 49 (1): 76-79.

[8] Chen Guodong GW. Current status of research on nausea and vomiting after general anesthesia $[\mathrm{J}]$. International Journal of Anesthesiology and Resuscitation, 2015; 36 (11).

[9] Zhou Chunyan YL, Fang Dingzhi, et al. Biochemistry and Molecular Biology (9th ed.) [M] [J]. Beijing: People's Medical Publishing House, 2018: 177-207.

[10] Berghe G, Van Den, Wouters P. Weekers F, et al. Intensive insulin therapy in critically ill patients $[\mathrm{J}]$. Chinese Critical Care Medicine, 2009; 18 (2): 1586-1588.

[11] Wang Zhiguo YW, Qin Huanlong, et al. Effects of preoperative oral carbohydrates on postoperative insulin resistance and its mechanism [J]. Chinese Journal of Basic and Clinical Medicine, 2008; 15 (11): 799-804.

[12] Henriksen MG, Hessov I, Dela F, et al. Effects of preoperative oral carbohydrates and peptides on postoperative endocrine response, mobilization, nutrition and muscle function in abdominal surgery $[\mathrm{J}]$. Acta Anaesthesiologica Scandinavica, 2010; 47 (2): 191-199. 
[13] Manchikanti L, Malla Y, Wargo BW, et al. Preoperative fasting before interventional techniques: is it necessary or evidence-based? [J]. Pain Physician, 2011; 14 (5): 459-467.

[14] Wu Bijing HX, Chen Bizhu. Clinical study on the effects of different fasting time before surgery on elective stress ulcer in patients undergoing elective surgery $[\mathrm{J}]$. Zhongguo Medical Journal, 2014 (9): 6-7.
[15] Boron WF, Boulpaep EL. Medical Physiology: A Cellular And Molecular Approach [J]. 2009.

[16] Wang Tingwei LZ, Shen Linlin, et al. Physiology (9th ed.) [M] [J]. Beijing: People's Medical Publishing House, 2018; 177-207: 177-207.

[17] Canada NL, Mullins L, Pearo B, et al. Optimizing Perioperative Nutrition in Pediatric Populations [J]. Nutrition in Clinical Practice, 2016; 31 (1): 49-58. 